\title{
Über das Fett des Blutes bei gesunden und kranken Pferden.
}

\author{
von
}

Tierarzt Johann Rudolf, k. u. k. Mil.-Unt.-Tierarzt.

Aus dem Institut für medizinische Chemie der tierärztlichen Hochschule in Wien.

(Der Redaktion zugegangen am 7. Oktober 1917.)

Wenn ich mit der folgenden Studie ein Gebiet betrete, welches bisher so gut wie unbebaut ist, so bin ich mir wohl bewußt, daß es kaum möglich sein wird, die im ersten Ansturm gewonnenen Resultate so zu überblicken, daß jedes seine jeden Zweifel ausschließende Erklärung finden müßte. Trotz des großen Interesses, welches die chemische Zusammensetzung der fettartigen und lipoiden Substanzen derzeit in der Biologie und Pathologie findet, ist nicht einmal die naheliegende Frage, wie aus dem Fette der Nahrung das Körperfett wird, soweit geklärt, als es wünschenswert wäre.

Wenn ich nun trotz der mangelnden Voraussetzungen für eine richtige Beurteilung der Ergebnisse den Versuch unternommen habe, Fett und Lipoide des Blutes gesunder Pferde mit denen des Blutes von kranken zu vergleichen, so geschah dies hauptsächlich in dem Bestreben, zu erfahren, ob in diesem Punkte Krankheiten überhaupt Veränderungen hervorrufen, d. h. $o b$ das von mir betretene Gebiet überhaupt bebauungsfähig ist. Da viele Krankheiten, auch solche lokaler Natur mit Allgemeinerscheinungen, insbesondere Fieber, einhergehen, so ist es klar, daß bei diesen Krankheiten auch der Stoffwechsel verändert ist. Die allbekannte Tatsache, daß bei solchen Krankheiten auch die Fettpolster schwinden und zwar mitunter rascher, als durch die verminderte Nahrungsaufnahme erklärt werden kann, macht es von vornherein wahrscheinlich, da $B$ auch die Fette an den erwähnten Stoffwechselstörungen beteiligt sind.

Der Grund, warum ich zunächst im Blute Véränderungen der Fettsubstanz suchte, lag darin, daß einerseits das Blut bei Lebzeiten der Tiere gewonnen, auch in Zwischenräumen von einigen Tagen mehrmals demselben Tiere entnommen 
werden kann und daß somit kontrolliert werden kann, ob die Veränderungen der Fettsubstanzen mit dem Fortschreiten oder Abllauen der pathologischen Prozesse gleichen Schritt halten oder nicht. Anderseits würde ein anderes Objekt, beispielsweise das Körperfetl, erfordern, daß auch dessen absolute Menge in Rechnung gezogen wird, was in der praktischen Durchführung gewissen Schwierigkeiten begegnet.

Allerdings tauchen beim Blute wieder Schwierigkeiten anderer Natur auf. Das Blut, welches die Aufgabe hat, Stoffe von einem Organ des Körpers zu einem anderen zu transportieren, kann in dieser Beziehung mit einem Eisenbahnzuge verglichen werden. Bei einem Eisenbahnzuge ist aber wohl zu unterscheiden $z$ wischen dem Materiale, aus welchem der leere Zug besteht, und dem Materiale, welches auf dem Eisenbahnzuge verladen wurde. Der leere Eisenbahnzug wäre etwa dem vergleichbar, was ich als «Blut an sich * bezeichnen möchte, während die Ladung aus jenen Stoffen bestehen würde, welche das Blut eben zu transportieren hat.

Zur chemischen Untersuchung bekommt man aber beide Gruppen von Stoffen gemischt und es muß erst durch nachträgliche Überlegung ermittelt werden, wieviel von den Resultaten auf das "Blut an sich " und wieviel auf die sLadung" zu rechnen wäre.

Bei oberflächlicher Betrachtung könnte ein einfacher Weg zur Darstellung des «Blutes an sich» darin gegeben scheinen, daß man das Blut im Hungerzustande untersucht. Abgesehen davon, daß dieser Weg bei kranken Pferden nicht praktisch wäre, bildet der Hungerzustand keinen normalen Zustand und wie sich später ergeben wird, sind gerade die lipoiden Stoffe des "Blutes an sich» von Einflüssen stark abhängig.

Ich glaubte aber aus dieser Schwierigkeit durch folgende Überlegung herausfinden zu können, die, wenn sie auch nicht in allen Teilen vollauf bewiesen ist, doch für eine Studie, welche wie vorliegende nur orientierenden Charakter haben soll, ausreichend Anhaltspunkte zu bieten scheint.

Was das Blutplasma betrifft, so kann dessen Gehalt an Fettsubstanzen wohl kaum auf das *Blut an sich» bezogen 
Ober das Fell des Blutes bei gesunden und kranken Pferden. 101

werden, seine Fettsubstanzen werden wohl ganz oder nahezu vollständig als "Ladung" zu deuten sein. Die Fettsubstanzen der roten Blutkörperchen bestehen wesentlich aus Cholesterin, während sie kaum Neutralfett enthalten. Demnach wäre unter den Fettsubstanzen des Blutes das Cholesterin der Hauptrepräsentant für das "Blut an sich», während das Neutralfett als Ladung aufzufassen wäre. Veränderungen, welche der Cholesteringehalt bei Krankheiten erleidet, beträfen demnach das «Blut an sich», während Veränderungen des Neutralfettgehaltes der «Ladung anzurechnen wären.

Demnach erschien meine Aufgabe in zwei Teile geteilt, von denen der eine das Neutralfett, der andere das Cholesterin des Blutes betrifft. Der erstere Teil der Aufgabe, der sich auf das Neutralfett bezieht, berührt sich mit jenen Fragen, welche in der Literatur sich um das Schlagwort «Lipämie. gruppieren, und es sei mir daher gestaltet, den gegenwärtigen Stand dieser Frage kurz zu skizzieren.

Unter Lipämie versteht man allgemein das Vorkommen von Fetttröpfchen im Blute. Daß ein Transport von Fett im Tierkörper noch in anderer Weise als in Form von Fetttröpfchen stattinden kann, beweisen die Untersuchungen von T. Miescher (1877) am hungernden Lachs, bei welchem das Blutserum völlig klar und durchsichtig ist, obwohl auf histo-chemischen Wege eine Fettwanderung konstatiert werden konnte.

Die Lipämie pllegt man in eine physiologische und eine pathologische einzuteilen.

Die physiologische Lipämie, welche man auch Verdauungs- oder Mastlipämie nennen könnte, tritt regelmäßig nach fettreichen Mahlzeiten auf und verschwindet einige Zeit nach der Mahlzeit wieder. Sie dauert eben nur so lange, als der Ductus thoracicus dem Blute mehr Felt zuführt, als dieses Organ bezw. Fettdepot normal abzugeben vermag.

Die pathologische Lipämie ist zwar in ihrer Pathogenese noch nicht genügend geklärt, es scheint aber sicher zu sein, daß sie nichts Einheitliches darstellt. So findet sie sich bei verschiedenen Krankheiten, ja selbst bei Aderlässen, wie Untersuchungen von Boggs und Morris (1908) sowie Pratt und 
Morawitz zoigon, ist man imstando, eine lipümis: zu erzougon. Auch Gifto kïmen cine solcho hervorrufen, wie dies insbesondero beim Phonylhydruzin uachgewiesen ist.

Fïr die lintstrhung dor pathologischen liplimie können liaktoren verschiodener Ait veruntwortlich gemacht werden. Sciner Ilerkunft nach kann das die Lipämic bedingende Fett zweierloi Art noin.

1. lis kann listl sein, wolches aus der Nahrung resorbiert wurde, also exogen in das Blut kum und durch krankhafte Verhithisse vortibergehend oder viclleicht sogar dituernd im Blute verwoilt.

2. Das Blutfelt stamml nicht vom Nalirungsfett, sondern vom Körperfell, scin Vorkommen im lllute ist also endogener Natur und hängt mil cinor follwanderung zusammen.

Aulser der Provenienz des Blulfelles kominen ferner bei der kranklıafton Lipümic noch Störungen der Abgabe des feltes atus dorn Blute in Betracht. Man hat von einem relativen Unvermïgen des Blutes gesprochen, das Fell in der normalen \%eil wieder abrugoben, und hat die Ursache dieses Unvermügens im Blute sclbst, in den Kapillaren und in den Geweben gesucht.

In Blute würde die Ursache golegen sein, wemn dem Blute die Fïligkeit teilwoise oder ganz inangelte, das Felt in jenen \%ustand überzulühren, der es befähigl, die Gefiilßwandung \%u durchwindern.

Pflügor glaubl, daß normalerweiso im Blute ein fettspaltendes Enzym, cine Lipaso oder Esterase, enthalten sei, welches den Fettiustritt. sus dem Blute beherrscht.

Dio Kapillaron könnten dadurch zur Ursache einer mangelnden Feltausscheidung aus dem Blute werden, daß ihre Wandung fiir don Durchtritt von Fetl weniger durchlässig oder undurchltissig wird, und ihr Gewebe dadurch, dals seine Zellen die Fühigkeil, Felt an sich zu reißen, verlieren.

Das Futter, welches die Pferde, deren Blut zurr Untersucluung golangle, erhielten, war boi allen Pferden qualitativ dasselbe; es bestand aus Haler, Heu und Strohhäcksel. Quantitativ ergaben sich nur darin Vorschiedenheilen, daß lranke Pferde nicht das ganze vorgelegte Fulter aulgenommen hatten. 
Über das Fett des Blutes bei gesunden und kranken Pferden. 103

Das Futter, welches die Tiere erhielten, muß wohl in seiner Gesamtheit als fettarmes Futter betrachtet und sein Einfluß auf den Gehalt des Blutes an Neutralfett also gering geschätzt werden." Er kommt zum Ausdrucke in der Menge und Besehaffenheit des Neutralfettes, welches im Blute gesunder Pferde enthalten ist. Stimmt nur die Jodzahl des Neutralfettes im Blute eines kranken Pferdes mit der Jodzahl, welche bei gesunden Pferden ermittelt wurde, überein, so deutet das auf einen exogenen Ursprung des Fettes hin; weicht sie aber erheblich davon ab, so macht diese Abweichung einen endogenen Ursprung des Fettes wahrscheinlich.

Wie sich aus den späteren Ausführungen ergeben wird, beziehen sich meine Betrachtungen über das Neutralfett vorzugsweise auf die Fettsäuren derselben. $\mathrm{Zu}$ diesem Zusammenhange können wohl die Phosphatide des Blutes unter das Neutralfett subsumiert werden.

Was die zweite Gruppe der von mir behandelten Frage betrifft, so kommen dafür jene Literaturangaben in Betracht, die sich auf das Cholesterin beziehen. Jener recht umfangreiche Teil der Literatur, der sich mit der chemischen Konstitution des Cholesterins befaßt, kann hier füglich übergangen werden. Es interessiert hier nur die biologische Seite.

Die früher allgemein geltende Ansicht, daß das Cholesterin eine biologisch bedeutungslose, gewissermaßen nur als Abfallsprodukt zu deutende Substanz sei, kann heute als widerlegt gelten. Wenn wir auch nicht die ganze biologische Bedeutung des Cholesterins erfassen können, so steht doch jedenfalls fest, daß das Cholesterin sich hervorragend an der Bildung der Zellmembranen der tierischen Zellen, insbesondere der roten Blutkörperchen, beteiligt und somit die biochemischen Prozesse in der Zelle beeinflußt und daß das Cholesterin eine antitoxisch wirkende Substanz ist.

In den letzten Jahren hat man begonnen, sich intensiver mit dem Cholesterinstoffwechsel zu beschäftigen. Diese Arbeiten haben indessen noch nicht zu vollkommen geklärten Vorstellungen über den Cholesterinstoffwechsel geführt. Sicher und längst bekannt ist, daß Cholesterin mit der Galle in den Darm aus- 
geschieden wird. $\mathrm{Ob}$ aber auch eine Ausscheidung des Cholesterins auf einem anderen Wege erfolgt, ist nicht bekannt, speziell auch nicht, ob die ihrer chemischen Konstitution nach dem Cholesterin verwandten Gallensäuren mit dem Cholesterin auch genetisch zusammenhängen.

Daß übrigens verschiedene Tiergattungen bei der Ausscheidung des Cholesterins sich verschieden verhalten, geht aus den Untersuchungen von 0 . Weltmann hervor, welcher nachweisen konnte, daß Pflanzenfresser (Kaninchen und Meerschweinchen) zugeführtes Cholesterin aufstapeln, während Fleischfresser (Hund und Katze) dies nicht tun.

Aus den Arbeiten von Bacmeister und Haves( $\left.{ }^{1}\right)$ ergibt sich, daß die Menge des jeweilig in der Galle ausgeschiedenen Cholesterins parallel geht mit dem Cholesteringehalt des Blutes.

Recht zweifelhaft ist aber die Herkunft des Cholesterins. Außer Zweifel steht, daß vom Fleischfresser typisches, tierisches Cholesterin mit der Nahrung aufgenommen wird, indem dieses typische, tierische Cholesterin bei allen Wirbeltiergattungen bis zu den Protozoen herunter als identisch befunden wurde. Etwas schwieriger ist die Beurteilung dieser Frage beim Pflanzenfresser, da die verschiedenen pflanzlichen Cholesterine (Phytocholesterine) zwar dem tierischen Cholesterin nahe verwandt, aber nicht mit ihm identisch sind, daher im Organismus des Pflanzenfressers erst zu tierischem Cholesterin umgewandelt werden mußten. Recht kompliziert wird die Frage dadurch, daß im Pflanzenreiche recht verbreitete Farbstoffe vorkommen, die Carotine, welche dem Cholesterin mehr oder minder verwandt sind, und daß auch im Tierkörper regelmäßig Stoffe vorkommen, welche dem Cholesterin ihrer Konstitution nach und teilweise zweifellos auch genetisch nahestehen. Hierher gehören die Oxycholesterine und die Lipochrome. Ist es schon zweifelhaft, ob dem Cholesterin verwandte Stoffe im Tierkörper zu Cholesterinen umgebildet werden können, so liegt noch völliges Dunkel über der Frage, ob der Tierkörper Cholesterin frei aufbauen kann.

Der Cholesterinbestand des Tierkörpers hängt übrigens gewiß nicht allein von der Zufuhr ab. Insbesondere sind Krank- 
Uber das Felt des Blutes bei gesunden und kranken Pferden. 105

heitsprozesse darauf von Einfluß. Längst bekannt ist das Vorkommen von Cholesterin in Cysten. Auch bei degenerativen Vorgängen spielt das Cholesterin, wie Panzer( $\left.{ }^{2}\right)$ auf chemischem und $A s \operatorname{choff}^{3}$ ) auf histologischem Wege nachgewiesen haben, eine Rolle und zwar so sehr, daß Aschoff eine Cholesterinvon einer Glycerinverfettung unterscheidet.

Daraus geht hervor, daß der Cholesteringehalt des Blutes von den verschiedensten Einflüssen abhängig sein wird. Direkt nachgewiesen wurden Änderungen des Cholesteringehaltes des Blutes (und der Galle) in der oben angeführten Arbeit von Bacmeister und Haves. Sie fanden eine Vermehrung der Cholesterine

a) bei reichlicher Nahrung,

b) besonders bei fett- und eiweißreicher Nahrung,

c) bei Wöchnerinnen unmittelbar vor der Geburt.

Eine Verminderung wurde festgestellt:

a) bei mangelhafter Nahrungszufuhr,

b) bei allen fieberhaften Krankheiten,

c) im letzten Drittel der Schwangerschaft.

Speziell mit dem Cholesteringehalt des menschlichen Blutes bei inneren Krankheiten beschäftigt sich eine Arbeit von E. Henes, (4) welcher bei schweren Stoffwechselerkrankungen, nämlich Diabetes, Fettsucht, Nephritis und frischer Arteriensklerose eine Hypercholesteriämie fand, während bei schlechtem Allgemeinbefinden, Fieber, Alter und entsprechender Diät der Cholesteringehalt des Blutes sinkt. Derselbe Autor kommt auf Grund seiner Untersuchungen zu dem Schlusse, daß das Cholesterin nicht im Körper gebildet wird, sondern daß das im Körper zu findende Cholesterin aus der Nahrung sei.

Die zuletzt zitierte Arbeit bildete die unmittelbare Veranlassung $\mathrm{zu}$ der im folgenden beschriebenen Untérsuchung. Auch beim Cholesterin beziehen sich die bisherigen Arbeiten auf den Menschen und die gewöhnlichen kleinen Versuchstiere. Entsprechende Untersuchungen am Pferde liefern vor allem deshalb größere Aussichten, weil es beim Pferde durch einen für das Allgemeinbefinden des Pferdes bedeutungslosen Aderla $B$ unschwer möglich ist, solche Quantitäten Blut zu gewinnen, 
daß einerseits die gewöhnlichen bewährten Untersuchungsmethoden angewandt, anderseits mit dem Blut von einem Aderlasse mehrere quantitative Bestimmungen vorgenommen werden können. Ferner ist das Pferd an eine ständige Nahrung gewöhnt, nämlich an Heu, Hafer, Strohhäcksel; es lassen sich dadurch leicht die Einflüsse der Nahrung ausschalten bezw. konstant erhalten.

Endlich beanspruchen die Untersuchungen am Pferd auch dadurch Interesse, daß das Pferd als Pflanzenfresser, wie oben angeführt wurde, mit der Nahrung kein typisches, tierisches Cholesterin aufnimmt, folglich seinen Cholesteringehalt selbst bilden muß, wofür ihm allerdings nehr oder minder nahestehende Stoffe (Phytosterin) zur Verfügung stehen. Schon aus diesem Grunde ergibt sich, daß der oben erwähnte Schluß von E. He nes im strengsten Sinne nicht allgemein zutreffend sein kann.

\section{Methodik.}

Nach den vorstehenden Ausführungen war also die Aufgabe gestellt, den Gehalt des Pferdeblutes an Cholesterin und Neutralfett zu bestimmen, sowie das Neutralfett durch die Bestimmung der Jodzahl zu charakterisieren.

Bei der Auswahl geeigneter Methoden zur Durchführung dieses Planes konnte ich mich durch umfangreiche Voruntersuchungen überzeugen, daß die Behandlung des auf die eine oder auf die andere Art zur Trockene gebrachten Blutes mit fettlösenden Flüssigkeiten die Fettsubstanzen nur unvollständig dem Blute entzieht. Als zweckmäßigste Methode ergab sich die von Kumagava-Suto in der von Shimidzu(5) für das Blut ausgearbeiteten Modifikation.

Ohne auf das Detail der Methode und auf von mir vorgenommene Abänderungen in der Apparatur, die sich.als praktisch erwiesen haben, einzugehen, sind die Grundlagen der Methode folgende.

Die gemessene Blutmenge wird mit 95\% igem Alkohol ausgefällt und das entstandene Koagulum andauernd mit heißem Alkohol extrahiert. Die vereinigten alkoholischen Auszüge werden mit Ätznatron versetzt und bis zur vollständigen Verseifung des Neutralfettes gekocht, wobei ich die Bedingungen 
Über das Fett des Blutes bei gesunden und kranken Pferden. 107

so wählte, daß auch die Cholesterinester, deren Vorkommen im Blute durch Hürthle nachgewiesen ist, verseift wurden. Nach Verdunstung des Alkohols wird der Rückstand mit Wasser aufgenommen, durch Zusatz von Salzsäure die Seifen zerlegt und die Flüssigkeit mit Äther ausgezogen. Die in den Äther übergegangenen Stoffe werden nach dem Verjagen des Äthers mit Petroläther aufgenommen, die Lösung filtriert und verdunstet. Der Verdunstungsrückstand besteht aus den gesamten im Wasser unlöslichen Fettsäuren, welche im Blute in Form von Neutralfett, Cholesterinestern oder Seifen enthalten waren, und dem sogenannten unverseifbaren Rücktande.

Dieser unverseifbare Rückstand setzt sich zusammen aus dem gesamten Cholesterin des Blutes (freies Cholesterin und Cholesterine aus den Cholesterinestern) und anderen im Petroläther löslichen Substanzen von nicht saurer Natur, beim Blute jedenfalls höhere Alkohole von nicht näher bekannter Konstitution (darunter auch Oxycholesterine).

Dieser Verdunstungsrückstand wurde gewogen und zu weiteren Untersuchungen verwendet.

Die beschriebene Methode hat den großen Vorteil, daß sie mit relativ kleinen Blutmengen durchgeführt werden kann und daß sie insofern genau arbeitet, als die Gewichte des Petrolätherextraktes von drei Blutproben desselben Aderlasses stets gut übereinstimmen. Für eine Untersuchung entnahm ich im Stalle den Pferden das Blut aus der Vena jugularis mit einer Hohlnadel und fing es in einem bereitgehaltenen Becherglase auf. Von diesem Blute wurden sofort mit der Pipette drei Proben zu je $10 \mathrm{ccm}$ entnommen, in je $50 \mathrm{ccm} 95 \%$ igen Alkohol gebracht und in der angedeuteten Weise zu Petrolätherextrakt verarbeitet.

Weitere Proben des Blutes wurden nach der üblichen. Methode zur Bestimmung des spezifischen Gewichtes auf pyknometrischen Wege verwendet.

Die drei Petrolätherextrakte aus je $10 \mathrm{ccm}$ Blut, deren Gewichte, wie schon erwähnt, gut übereinstimmten, wurden weiter wie folgt verarbeitet.

Die erste Probe diente zur Bestimmung der Jodzahl des Gesamtpetrolextraktes nach Hübl. 
Die zweite Probe wurde zur Bestimmung der Menge der unverseifbaren Substanz, nachdem die Fettsäuren durch Kalilauge gebunden worden waren, verwendet. An dem unverseifbaren Rückstande wurde, nachdem er gewogen war, wieder die Jodzahl bestimmt.

In der dritten Probe wurde das Cholesterin nach der Digitoninmethode nach Windaus( $\left(^{(}\right)$quantitativ bestimmt.

Damit waren direkt bestimmt:

1. Das spezifische Gewicht.

2. Die Trockensubstanz.

3. Gesamtpetrolätherextrakt.

4. Der unverseif bare Rückstand.

5. Das Cholesterin.

6. Die Jodzahl des Gesamtpetrolätherextrakles.

7. Die Jodzahl des unverseifbaren Rückstandes.

Aus diesen direkt bestimmten Daten konnten durch Rechnung ermittelt werden:

8. Die wasserunlöslichen Fettsäuren (aus 3 und 4).

9. Das Neutralfett aus 8 mit Hilfe einer Konstanten.

10. Der nicht aus Cholesterin bestehende Anteil des unverseifbaren Rückstandes: Andere unverseifbare Substanzen (aus 4 und 5).

11. Die Jodzahl der Fettsäuren (aus 3, 4, 6, 7 und 8). Es wäre gewiß recht interessant gewesen, näheres über die chemische Natur der Substanzen zu erfahren, welche unter dem Titel: «Andere unverseifbare Substanzen» quantitativ bebestimmt wurden, und deren Menge, wie die folgenden Tabellen zeigen, gar nicht so gering ist. Einen Anhaltspunkt dafür hätte ihre Jodzahl geben können. Ich habe es aber unterlassen, diese Jodzahl aus den gegebenen Daten und der bekannten Jodzahl herauszurechnen. Es schien mir diese rechnerische Ermittelung nicht verläßlich genug, weil sich auf diese Zahl zu viele unvermeidliche Versuchsfehler häufen.

Was die Ermittelung der Menge des Neutralfettes betrifft, so wird gewiß nicht verkannt, daß die bezügliche Zahl jedenfalls nicht dem wahren Wert entspricht. Man hat sich aber in Erkenntnis der Unzulänglichkeit der direkten Bestimmungs- 
Über das Fett des Blutes bei gesunden und kranken Pferden. 109

methoden vielfach auch anderwärts damit abgefunden, die verläßlichere bestimmbare Menge des Neutralfettes zu verwenden und damit, wenn auch nicht $\mathrm{zu}$ absolut richtigen, so doch wenigstens zu vergleichbaren Werten zu gelangen.

Vergleichsweise kamen auch einige Proben Körperfett zur Untersuchung. Zu diesem Behufe wurden aus dem Kadaver des Pferdes Fettgewebe ausgeschnitten und nach der Methode der direkten Verseifung nach Kumagava-Suto auf Petrolätherextrakt verarbeitet, welcher dann weiter untersucht wurde.

\section{Die zur Untersuchung verwendeten Pferde.}

Insgesamt kamen 46 Blutproben, eine Probe eines Pleuritisexsudates und sechs Proben von Körperfett zu der eben skizzierten Untersuchung, welche bei einigen Proben nicht in vollem Umfange durchgeführt wurde. Die Blutproben stammen von 32 Pferden, die in den Kliniken der Tierärztlichen Hochschule in Wien zur Behandlung eingestellt waren. Dieselben sind zumeist durch eine kurzes Nationale und durch eine ganz auszugsweise Krankheitsgeschichte charakterisiert. Die fortlaufenden Zahlen bedeuten gleichzeitig die im folgenden festgehaltenen Nummern der chemisch untersuchten Proben.

\section{Gesunde Pferde.}

1. P. Nr. 1010. Stichelhaariger Schweißfuchs, W., $4 \frac{1}{2}$ J. alt, gutgenährtes leichtes Wagenpferd.

2. P. Nr. XII. Lichtfuchs, W., 5\%/2 J. alt, ziemlich gut genährtes leichtes Wagenpferd.

3. P. Nr. 1485. Kastanienbraune St., 14 J. alt, leichtes, gut genährtes Wagenpferd.

4. P. Nr. 1600. Kastanienbrauner W., 5 J. alt, mittelschweres, gut genährtes Wagenpferd.

5. P. Nr. 1632. Dunkelfuchs, W., 5 J. alt, gut genährtes-Wagenpferd.

6. P. Nr. 1982. Kastanienbraune Stute, bei 19 J. alt, schlecht genährtes, leichtes Wagenpferd.

\section{Brustseuche.}

7. P. Nr. 1060 . Kastanienbrauner W., bei 16 J. alt, schweres Wagenpferd.

Diagnose: Pneumonia dextra. 
Das Pferd ist vor 13 Tagen der Klinik zugewachsen mit einer Temp. 40,6 ${ }^{\circ}$, Puls 60, Atem 20. - Bei der Blutentnahme, 13 Tage später, ist das Pferd beroits außer Behandlung; die FreBlust ist sehr gut.

8. P. Nr. 1158. Dunkelkastanienbrauner W., 9 J. alt.

Diagnose: Brustseuche, Pneumonia bilateralis.

Bei der Blutentnahme am 4. Tage nach der Einstellung in die Klinik hat es T. $40,3^{\circ}$, P. 68 , A. 22 . Dämpfung auf beiden Seiten vorhanden. Freßlust schlecht, trinkt viel Wasser. Matt, Ernährungszustand gat.

9. P. Nr. 1154. Kastanienbrauner Wall., 9 J. alt.

Diagnose: Pneumonia dextra, Petechialfieber und subpleurales Lungenemphysem.

Blutentnahme am 11. Tage nach dem Zuwachsen in die Klinik, T. 39,4 ${ }^{\circ}$, P. 90, A. 18. Dämpfung rechts nachweisbar. Das Tier ist stark abgemagert. Als Komplikation die charakteristischen Symptome des Petechialfiebers. Exspirationsluft übelriechend. Das Tier hat einen starken Durchfall.

10. P. Nr. 1158. Zum 2. Male 10 Tage später untersucht. Links ist die Dämpfung noch nachweisbar. T. 37,6, P. 36, A. 20. Das Tier ist während der 10 Tage stark abgemagert; Freßlust sehr gut.

11. P. Nr. 1433. Rotschimmel, W., 4 J. alt, schweres Zugpferd. Diagnose: Brustseuche, Pneumonia dextra.

Die Blutentnahme am 5. Tage der Erkrankung. T. 40, P. 70, A. 24. Dämpfung rechts nachweisbar. Das Tier ist matt, Futter- und Getränkaufnahme schlecht. Futter schlecht verdaut. Einen Tag vor der Blutentnahme hat das Tier Neosalvarsan bekommen.

12. P. Nr. 1433. Zum zweiten Male untersucht 6 Tage später. Pferd außer Behandlung.

13. P. Nr. 1080.

Diagnose: Brustseuche, Pneumonia bilateralis exsudativa.

Die Blutentnahme am 34. Tage der Erkrankung. T. $38,4^{\circ}$, P. 40 , A. 20. Dämpfung beiderseits über die Mitte des Brustkorbes reichend. Allgemeinbefinden schlecht. Zweimal punktiert und gegen 30 l Exsudat entleert. Es scheint, daß das Pferd der Besserung entgegengeht.

14. P. Nr. 1040. Kastanienbrauner Wall., 5 J. alt.

Diagnose: Brustseuche, Pneumonia bilateralis exsudativa.

Das Pferd steht seit 33 Tagen an der Klinik zur Behandlung. T. $39,8^{\circ}$, P. 62, A. 20. Dämpfung auf beiden Seiten bis handbreit über dem Buggelenke nachweisbar. Das Tier ist stark abgemagert und nimmt kein Futter auf. Schwellungen an der Unterbrust. Zweimal Exsudat in der Menge von 201 entnommen.

15. P. Nr. 1040. Untersuchung des Exsudates, am 36. Tage der Erkrankung entnommen. Krankeitssymptome dieselben, wie bei der ersten Blutentnahme. Freßlust hat sich etwas gebessert.

16. P. Nr. 1040. Zweite Blutenlnahme $24^{\mathrm{h}}$ vor dem Tode. T. $39,3^{\circ}$, P. 72, A. 24. 
Über das Fett des Blutes bei gesunden und kranken Pferden. 111

17. P. Nr. $1433=$ Nr. 12.

18. P. Nr. 1505. Kastbrauner W., 5 J. alt, leichtes Wagenpferd.

Diagnose: Brustseuche, Pneumonia dextra, Pleuritis, Angina.

Die.Blutentnahme am 4. Tage nach der Aufnahme in die Klinik. T. $40,1^{\circ}$, P. 30, A. 16. Dämpfung rechts handbreit über das Ellbogengelenk. Reibegeräusche.

19. P. Nr. 1557. Kastanienbr. W., $4^{1 / 2}$ J. alt, gut genährtes, mittelschweres Wagenpferd.

Diagnose: Brustseuche.

Blutentnahme am 3. Tage der Erkrankung, nachdem es zwei Tage vorher Neosalvarsan bekommen hat. T. 39,4ํ, P. 52, A. 32. In der Lunge nichts nachweisbar. Das Pferd ist matt.

20. P. Nr. 1557. Zweite Blutentnahme drei Tage später. Geringgradiger Ikterus. T. $38,6^{\circ}$, P. 48, A. 24. Appetit gut.

21. P. Nr. 1704. Lichtbrauner W., 7 J. alt, gut genährtes schweres Wagenpferd.

Diagnose: Brustseuche mit Pneumonia centralis.

Das Blut am 5. Tage der Erkrankung entnommen. T. 39,4, P. 68, A. 32; starker Ikterus, verminderte Getränk- und Futteraufnahme.

22. P. Nr. 1704. Zweite Blutnahme.

Diagnose: Pneumonia catarrhalis.

Die Blutentnahme 7 Tage später. T. 40,4 ${ }^{\circ}$, P. 56, A. 20. Die Krankheitserscheinungen sprechen jetzt für eine Pneumonia catarrhalis.

23. P. Nr. 1704. Zum dritten Male untersucht.

Diagnose: Brustseuche, Pneumonia bilateralis.

Blutentnahme am 11. Tage der Erkrankung. T. 38, $4^{\circ}$, P. 48, A. 22; Dämpfung ist jetzt beiderseits nachzuweisen.

24. P. Nr. 1704. Vierte Blutentnahme am 25. Tage der Erkrankung. Das Pferd ist außer Behandlung.

25. P. Nr.1683. Sommerrapp, W., 6 J. alt, mittelschweres Wagenpferd.

Diagnose: Pneumonia sinistra, Gastroenteritis, Brustseuche.

Blutentnahme am 6. Tage der Erkrankung. T. 40,20, P. 48, A. 24 . Dämpfung links nachweisbar.

26. P. Nr. 1683. Zweite Blutentnahme. Am 9. Tage der Erkrankung Dämpfung kleiner geworden. Allgemeinbefinden gut.

27. P. Nr. 1683. Dritte Blutentnahme. Drei Tage nach nach der zweiten Entnahme durchgeführt. Dämpfung verschwunden. T. $37,5^{\circ}$, P. 40, A. 20. Futteraufnahme sehr gut.

28. P. Nr. 1683. Vierte Blutentnahme. T. $38,5^{\circ}$, P. 36, A. 20. Das Pferd ist außer Behandlung.

29. P. Nr. 1613. Kastanienbrauner W., 9 J. alt, schweres Wagenpferd.

Diagnose: Brustseuche, Pneumonia dextra.

Blutentnahme am 9. Tage der Erkrankung. T. $39,6^{\circ}$, P. 72, A. 30. Dämpfung rechts in der Höhe des Buggelenkes nachweisbar. 
30. P. Nr. 1817. Kastanienbrauner W., $10 \mathrm{~J}$. alt, schweres, gut gonăhrtes Wagenpferd..

Diagnose: Pneumonia catarrhal. Gastroenteritis.

Das Blut wurde unmittelbar vor dem Tode des Tieres entnommen. Bofund: 1/. Stunde vor dem Tode war T.39,2, P.92, A. 64. Blatiger NasenausfluB, Lungenbefund normal, Bauchumfang vergröGert. Aus dem Maule entleert sich eine stinkende Flüssigkeit. Kot ist breiig-flüssig, von üblem Geruch.

Sektionsbefand: Pneumonia catarrh. chron. Gastritis, Dilatatio ventriculi acuta, sowie akuter Darmkartarrh.

31. P. Nr. 1887. Stichelhaariger Lichtkastanienbraun., 16 J. alt, schweres, gut genährtes Wagenpferd.

Diagnose: Brustseuche, Pneumonia bilateralis.

Das Blut wurde am ersten Tage der Einstellung in die Klinik entnommen. T. $40,7^{\circ}$, P. 80, A. 40. Beiderseitige Dämpfung in der Höhe des Buggelenkes nachweisbar.

32. P. Nr. 1887. Blutentnahme zum 2. Male. Drei Tage später entnommen. Das Allgemeinbefinden des Tieres hat sich gebessert. Die Dämpfung besteht noch ungeändert. Freßlust ist ziemlich gut.

33. P. Nr. 1900. Schwarzbrauner W., 6 J. alt, mittelschweres Wagenpferd.

Diagnose: Pneumonia sinistra.

Bei der Blutentnahme ist die Pneumonie schon im Lösungsstadium. T. $38,8^{\circ}$, P. 60 , A. 26 . Allgemeinbefinden gut.

34. P. Nr. 1813. Kastanienbraune St., 2 J. alt.

Diagnose: Pleuritis, Petechialfieber, Gastroenteritis.

Blutentnahme am 16. Tage der Erkrankung. T. $40,0^{\circ}$, P. 80, A. 32.

35. P. Nr. 1887. Blutentnahme zum 3. Male. T. $38,7^{\circ}$, P. 60, A. 24. Allgemeinbefinden ist gut. Die Dämpfung beiderseits beinahe ganz geschwunden.

\section{Petechialfieber.}

36. P. Nr. 1480. Stichelhaariger, kastanienbrauner W., 8 J. alt. Diagnose: Petechialfieber.

Die Blutentnahme erfolgte 30 Stunden vor dem Tode des Tieres. T. $38,4^{\circ}$, P. 70 , A. 16.

37. P. Nr. 1509. Kastanienbrauner Wall., 10J. alt, schweres Zugpferd.

Diagnose : Petechialfieber.

Die Blutentnahme am 5. Tage der Erkrankung, T. 38,0, P. 60, A. 12. Charakteristische Krankheitserscheinungen des Petechialfiebers (Nilpferdkopf). Das Tier nimmt Futter auf. -

38. P. Nr. 1593. Dunkelkastanienbraune Stute, 9 J. alt, schweres, gutgenährtes Wagenpferd.

. Diagnose: Petechialfieber. 
Über das Fett des Blutes bei gesunden 'und kranken Pferden. 113

Blutentnahme am 10. Tage der Erkrankung, T. 39,8 , P. 60, A. 30. Die Schwellungen haben innerhalb der letzten 24 Stunden bedeutend zugenommen. 18 Stunden später ist das Tier erstickt.

Sektionsbefund: Pneumonia cruposa bilateralis im Stadium der grauen Hepatitation, Pleuritis dextra, Degeneration der Parenchyme.

39. P. Nr. 1743. Stichelhaariger, schwarzbrauner W., 6 J. alt.

Diagnose : Petechialfieber, chronisches Lungenemphysem.

Bei der Blutentnahme T. $38,0^{\circ}$, P. 36, A. 12 . Scheint auf dem Wege der Besserung zu sein.

40. P. Nr. 1779. Lichtbrauner W., bei $16 \mathrm{~J}$. alt, sehr schlecht genährtes Tier.

Diagnose: Petechialfieber.

Bei der Blutentnahme T. $39,0^{\circ}$, P. 80, A. 16.

41. P. Nr.1779. Zweite Blutentnahme 5 Tage später. Das Allgemeinbefinden des Tieres hat sich verschlechtert. Die Schwellungen haben zugenommen.

Sektionsbefund: Zahlreiche Blutungen in das Innere, Obstipatio der Arteria femoralis.

\section{Krankheiten des Verdaungstraktes.}

42. P. Nr. 1442. Dunkelfuchs, W., 6 J. alt, gut genährtes. schweres Zugpferd.

Diagnose: Gastroenteritis.

Blutentnahme am 4. Tage der Erkrankung. T. 38,0, P. 36, A. 12.

43. P. Nr. 1868. Kastanienbrauner W., 12 J. alt.

Diagnose: Gastroenteritis und Lungenemphysem.

Bei der Blutentnahme T. $38,0^{\circ}$, P. 50, A. 45. Schlechte Futteraufnahme und große Mattigkeit.

44. P. Nr. 1868. Zum zweiten Male. Blutentnahme am 10. Tage der Erkrankung. T. $39,7^{\circ}$, P. 80, A. 14.

45. P. Nr. 1885. Gemischter Schimmel, $10 \mathrm{~J}$. alt, kleines minder gut genährtes Wagenpferd.

Diagnose: Indigestion.

Das Blut wurde am 14. Krankheitstage entnommen. T. 38, $0^{\circ}$, P. 36, A. 12. Appetit gut. Der Kot ist breiig.

46. P. Nr. 2013. Kastanienbrauner W., bei 12 J. alt, schlecht genährtes leichtes Wagenpferd.

Diagnose: Gastroenteritis.

Blutentnahme am 7. Tage der Erkrankung, T. 38,0 , P. 48, A. 20. Keine Krankheitserscheinungen mehr nachweisbar.

47. P. Nr. 2068. Stichelhaariger Rotfuchs, 9 J. alt, gut genährtes, schweres Wagenpferd. 
Diagnose: Katarrhalischer Darmkrampf.

Die Blutentnahme geschah $1 / 4$ Stunde vor dem Verenden des Tieres. T. von 40,6 auf $39,9^{\circ}$ gefallen, P. 44, A. 72 . Starkes Muskelzittern am ganzen Körper, Schweißausbruch, Mand und Nase entströmt ein übler Geruch.

\section{Tetanus.} suchstieren.

Wurde nur ein einziger Fall untersucht, aus Mangel an Ver-

48. P. Nr. 15:27. Kastanienbrauner W., 7 J. alt, gat genährtes Wagenpferd.

Diagnose: Tetanus.

Blutprobe wurde am 6. Tage der Erkrankung entnommen. T. 39,0?, P. 52, A. 40. Das Tier nimmt noch etwas Futter auf.

\section{Körperfett.}

49. P. Nr. 1613. Kastanienbrauner W., 9 J. alt.

Diagnose: Brustseuche, Pneumonia dextra.

Von diesem Pferde wurde auch das Blut untersucht. (29.) Das Fettgewebe entstammt der Kniefalte und der Präputialgegend. Starke Abmagerung, daß beinahe das ganze Fett geschwunden war.

50. P. Nr. 1558. Klinische Diagnose: Pneumonia bilat.

Sektionsbefund: Pleuritis serofibrinosa bilateralis mit stellenweisen Adhäsionen in mittleren und unteren Abschnitten, gangränöse Pneumonie. Degeneration der großen Parenchyme.

51. P. Nr. 1570. Sektionsbefund: Gastroenteritis.

Zur Untersuchung wurde Kammfett verwendet.

52. P. Anatomiepferd.

Sektionsbefund: Brustseuche, Pneumonia sinistra.

Zur Untersuchung gelangte Kammfett. Das Tier ist stark abgemagert. Das Fett ist beinahe ganz geschwunden.

53. P. Nr. 1679. Klinische Diagnose: Tympanitis.

Sektionsbefund: Tympanitis.

54. P. Nr. 2068. Stichelhaariger Rotfuchs, $9 \mathrm{~J}$ alt.

Diagnose: Katarrhalischer Darmkrampf.

Wurde auch Blut untersucht. (47.) Das Fett stammte aus der Kammgegend.

\section{Direkt bestimmte Zahlen.}

Die im folgenden angeführten Gewichtsmengen in Gramm sind auf 1 Liter Blut (bezw. Exsudat) berechnet. 
Über das Fett des Blutes bei gesunden und kranken Pforden. 115

Gesunde Pferde.

\begin{tabular}{|c|c|c|c|c|c|c|c|c|}
\hline Nr. & $\begin{array}{c}\text { An- } \\
\text { merkungen }\end{array}$ & $\begin{array}{c}\text { Spezi- } \\
\text { fisches } \\
\text { Ge- } \\
\text { wicht }\end{array}$ & $\begin{array}{l}\text { Trocken- } \\
\text { substanz }\end{array}$ & $\begin{array}{l}\text { Ge- } \\
\text { samt- } \\
\text { petrol- } \\
\text { äther- } \\
\text { ex- } \\
\text { trakt }\end{array}$ & $\begin{array}{c}\text { Unver- } \\
\text { seif- } \\
\text { bare } \\
\text { Sub- } \\
\text { stanz }\end{array}$ & $\begin{array}{l}\text { Ghole- } \\
\text { sterin }\end{array}$ & $\begin{array}{l}\text { Jodzahl } \\
\text { des Ge- } \\
\text { samt- } \\
\text { petrol- } \\
\text { ätherex- } \\
\text { traktes }\end{array}$ & $\begin{array}{l}\text { Jodzahl } \\
\text { des un- } \\
\text { verseif- } \\
\text { baren } \\
\text { Rück- } \\
\text { standes }\end{array}$ \\
\hline 1 & & 1,0484 & 185,0 & 3,25 & 1,06 & - & 72,3 & 60,6 \\
\hline 2 & & - & - & 3,58 & 0,48 & - & 75,2 & 33,2 \\
\hline 3 & & 1,0415 & 196,4 & 3,80 & 1,30 & - & 73,2 & 48,3 \\
\hline 4 & & 1,0388 & 173,0 & 3,95 & 1,02 & 0,70 & 89,2 & 52,8 \\
\hline 5 & & 1,0506 & 200,0 & 3,66 & 1,44 & 0,60 & 78,8 & 54,6 \\
\hline 6 & & 1,0519 & 199,0 & 4,10 & 1,02 & 0,83 & 81,0 & 55,4 \\
\hline
\end{tabular}

Brustseuche.

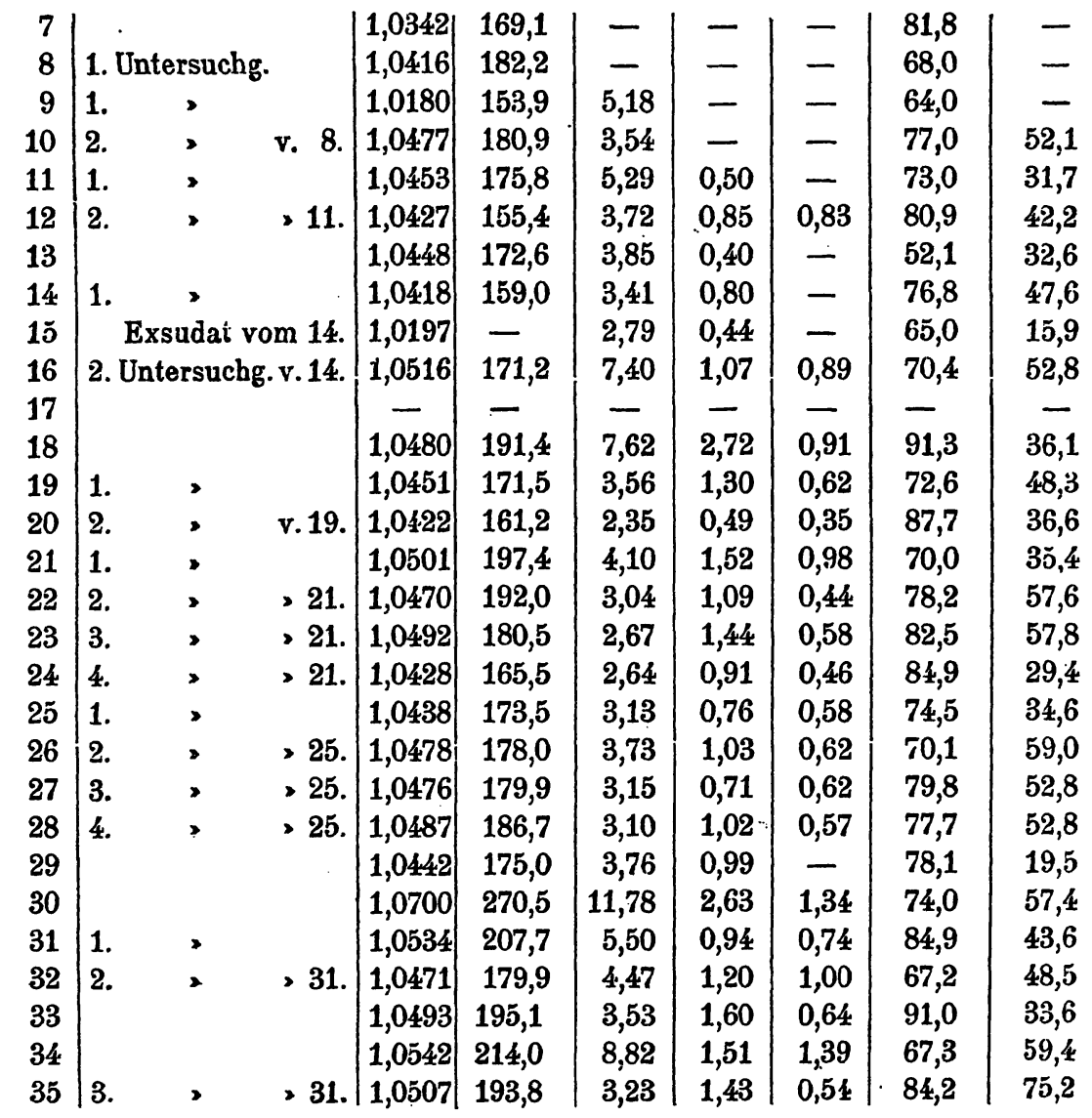


Petechialfieber.

\begin{tabular}{|c|c|c|c|c|c|c|c|c|}
\hline Nr. & Anmerkungen & $\begin{array}{l}\text { Spezi- } \\
\text { fisches } \\
\text { Ge- } \\
\text { wichl }\end{array}$ & $\begin{array}{l}\text { Trocken- } \\
\text { substanz }\end{array}$ & $\begin{array}{c}\text { Ge- } \\
\text { samt- } \\
\text { petrol- } \\
\text { äther- } \\
\text { ex- } \\
\text { trakt }\end{array}$ & $\begin{array}{l}\text { Unver- } \\
\text { seif- } \\
\text { bare } \\
\text { Sub- } \\
\text { stanz }\end{array}$ & $\begin{array}{l}\text { Chole- } \\
\text { sterin }\end{array}$ & $\begin{array}{l}\text { Jodzahl } \\
\text { des Ge- } \\
\text { samt- } \\
\text { petrol- } \\
\text { ätherex- } \\
\text { traktes }\end{array}$ & \begin{tabular}{|l} 
Jodzahl \\
des un- \\
verseif- \\
baren \\
Rück- \\
standes
\end{tabular} \\
\hline 36 & & 1,0664 & 215,4 & 4,64 & 1,27 & - & $8:, 5$ & 48,9 \\
\hline 37 & & 1,0502 & 199,7 & 4,32 & 0,97 & 0,71 & 72,2 & 50,4 \\
\hline 38 & & 1,0542 & 212,4 & 4,37 & 0,49 & - & 82,8 & 52,9 \\
\hline 39 & & 1,0488 & 189,4 & 3,98 & 0,99 & 0,49 & 78,1 & 52,8 \\
\hline 40 & & 1,0516 & 202.5 & 3,58 & 1,17 & 0,62 & 84,8 & 53,7 \\
\hline 41 & & 1,0360 & 136,8 & 3,42 & 0,90 & 0,47 & 84,1 & 59,7 \\
\hline
\end{tabular}

Krankheiten des Verdaungstraktes.

\begin{tabular}{|c|c|c|c|c|c|c|c|c|c|}
\hline 42 & \multirow{3}{*}{\multicolumn{2}{|c|}{ 1. Untersuchg. }} & $|1,0490|$ & 189,0 & 3,67 & 0,71 & - & 85.5 & 80,0 \\
\hline 43 & & & 1,0451 & 176,0 & 4,64 & 2,14 & 0,77 & 62,9 & 36,8 \\
\hline 14 & & & 1,0539 & 217,2 & 7,65 & 1,60 & 0,96 & 73,9 & 50 \\
\hline 45 & 2. & v. 43 & 1,0486 & 200,0 & 10,74 & 2,79 & 1,07 & 86,1 & 37,9 \\
\hline 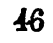 & & & 1,05 & 195,5 & 4 , & 2,07 & 0,95 & 79,4 & 56,3 \\
\hline 4 & & & $|1,0642|$ & 239,5 & 4,63 & 1,70 & 0,87 & 90,4 & 47,5 \\
\hline
\end{tabular}

$48 \mid$

Tetanus.

$|1,0616| 230,8|5,41| 1,80|1,04| 71,9$ | 33,3

Körperfett.

Die Resultate der Untersuchung von Körperfett sind so dargestellt, daß die Menge des Gesamtextraktes gleich 100 gesetzt wurde und die übrigen Zahlen auf die Menge bezogen werden.

Sämtliche unverseifbaren Rückstände addierten kein Jod, hatten daher die Jodzahl 0 und enthielten keine bestimmbare Menge an Cholesterin.

\begin{tabular}{c|c|c|c}
\hline \hline Nr. & Anmerkung & $\begin{array}{c}\text { Unverseif- } \\
\text { barer } \\
\text { Rückstand }\end{array}$ & $\begin{array}{c}\text { Jodzahl } \\
\text { des Gesamt- } \\
\text { extraktes }\end{array}$ \\
\hline 49 & von Nr. 29 & 1,57 & 46,3 \\
50 & & 1,01 & 51,9 \\
51 & - & 2,16 & 60,2 \\
52 & & 0,57 & 31,5 \\
53 & & 1,11 & 48,8 \\
54 & von Nr. 47 & 1,43 & 47,0
\end{tabular}




\section{Diskussion der Resultate.}

Für eine Diskussion der Resultate erscheint die obige tabellarische Zusammenstellung, welche nur die Originalzahlen umfaßt, nicht übersichtlich genug. Es werden daher im folgenden Tabellen angeführt, für welche durch Rechnung ermittelte Zahlen mit herangezogen, gleichfalls für 1 Liter Blut, bezw. beim Körperfett für Gesamtpetrolätherextrakt $=100$ berechnet. Die Tabellen umfassen die im zweiten Abschnitte unter $2,9,5,10,11$ und 7 näher erläuterten Werte.

\section{Gesunde Pferde.}

Den Proben $1-6$ sind in der folgenden Tabelle auch die Proben 12, 24 und 28 angefügt. Es sind dies Proben von bereits genesenen Pferden, also von Pferden, welche nach den Resultaten der klinischen Untersuchung als gesund betrachtet werden können.

\begin{tabular}{|c|c|c|c|c|c|c|c|}
\hline $\mathrm{Nr}$. & Anmerkung & $\begin{array}{c}\text { Trocken } \\
\text { substanz } \\
\text { in } g\end{array}$ & $\begin{array}{c}\text { Neu- } \\
\text { tralfett } \\
\text { in } g\end{array}$ & $\begin{array}{c}\text { Chole- } \\
\text { sterin } \\
\text { in } g\end{array}$ & $\begin{array}{c}\text { Andere } \\
\text { unver- } \\
\text { seifbare } \\
\text { Sub- } \\
\text { stanz } \\
\text { in } g\end{array}$ & $\begin{array}{c}\text { Jodzahl } \\
\text { der } \\
\text { Fett- } \\
\text { säuren }\end{array}$ & $\begin{array}{l}\text { Jodzahl } \\
\text { des un- } \\
\text { verseif- } \\
\text { baren } \\
\text { Rück- } \\
\text { standes }\end{array}$ \\
\hline 1 & & 185,0 & 2,29 & \multicolumn{2}{|c|}{1,06} & 78,4 & 60,6 \\
\hline 2 & & - & 3,24 & \multicolumn{2}{|c|}{1,30} & 81,7 & 33,2 \\
\hline 3 & & 196,4 & 2,62 & \multicolumn{2}{|c|}{0,48} & 86,2 & 48,3 \\
\hline 4 & & 173,0 & 3,06 & 0,70 & 0,32 & 101,9 & 52,8 \\
\hline 5 & & 200,2 & 2,82 & 0,60 & 0,74 & 88,2 & 54,6 \\
\hline 6 & & 199,2 & 4,22 & 0,83 & 0,19 & 89,4 & 55,4 \\
\hline 12 & จ. Pferde Nr. 11 & 155,4 & 3,00 & 0,83 & 0,02 & 92,9 & 42,6 \\
\hline 24 &,$\quad$, & 165,5 & 1,81 & 0,46 & 0,45 & 114,0 & 29,6 \\
\hline 28 & , , & 186,7 & 2,17 & 0,47 & 0,45 & 90,2 & 52.8 \\
\hline
\end{tabular}

Bei der Diskussion der vorstehend angeführten Werte sollen die der Blutproben 12, 24 und 28 nur mil einiger Vorsicht verwertet werden, weil ja von vornhinein nicht ausgeschlossen werden kann, da $\beta$ Veränderungen in den Fettsubstanzen des Blutes, welche mit der Krankheit einhergegangen sind, länger andauern, als die durch physikalische Untersuchung feststellbaren Symptome. 
Was zunächst die Menge des Neutralfettes betrifft, so wechselt sie allerdings ziemlich erheblich, wohl abhängig von der Zeit, welche zwischen der Fütterung der Tiere und der Entnahme der Blutprobe verstrichen ist. Wenn es auch nicht angezeigt ist, aus 6 bezw. 9 Einzelnwerten einen Durchschnittswert für den Fettgehalt des Blutes normaler Pferde zu ziehen, so wird dieser Durchschnittswert wohl etwa zwischen 2 und $4 \mathrm{~g}$ Neutralfett im Liter Blut zu suchen sein, und das genügt, um erhebliche Differenzen, welche im Blute kranker Tiere gefunden wurden, als abnormal bezeichnen zu können. Die Schwankungen in der Neutralfettmenge stehen in keinem Parallelismus mit der Gesamttrockensubstanz.

Wie schon früher erwähnt, wurde das Neutralfett durch die Jodzahl der Fettsäuren näher charakterisiert. Auch die hiefür gefundenen Zahlen differieren untereinander. Gar zu strenge wird man indes solche Differenzen nicht nehmen dürfen, weil diese Zahlen aus vier direkt bestimmten Werten berechnet sind, demnach gewissermaßen mit den vierfachen unvermeidlichen Versuchsfehlern behaftet sein können. Doch bewegen sich diese Zahlen innerhalb relativ enger Grenzen und zwar, wenn man nur die Proben $1-6$ in Betracht zieht, $z$ wischen 78 und 102.

Würde man sich eine ganz naive Vorstellung vom Fetttransport auf dem Wege der Blutbahn machen, dann würde man erwarten, daß die Jodzahl des Neutralfettes des Blutes zwischen der des Fettes des Futters und der des Körperfettes liegen mußte.

Für die letzteren geben die vorstehenden Zahlen für Körperfett einige Anhaltspunkte. Die Jodzahl der Fettsäuren berechnet sich daraus mit:

\begin{tabular}{|c|c|c|}
\hline Nr. & Jodzahl & der Fettsäuren \\
\hline 49 & & 47,0 \\
\hline 50 & & 52,4 \\
\hline 51 & & 61,5 \\
\hline 52 & $\cdots$ & 31,6 \\
\hline 53 & & 48,9 \\
\hline 54 & & 47,7 . \\
\hline
\end{tabular}


Über das Fett des Blutes bei gesunden und kranken Pferden. 119

also jedenfalls erheblich niedriger als beim Blute. Allerdings stammen die untersuchten Proben des Körperfettes nicht von gesunden, sondern von kranken Tieren. Im übrigen gibt die Literatur entsprechende Daten. Für das Pferdefett wird die Jodzahl der Fettsäuren angegeben

$$
\begin{aligned}
& \text { von } K a l m a n(7) \text { mit } 83-87,1, \\
& \text { von Anthor } u \text {. } Z \operatorname{Zink}\left({ }^{7}\right) \text { mit } 74,4-83,8 \text {. }
\end{aligned}
$$

Die Angaben der Literatur sind wesentlich höher als die von mir gefundenen Jodzahlen. Greife ich aus den letzteren die zwei niedrigsten, $\mathrm{Nr}$. 49 und $\mathrm{Nr}$. 52, heraus, so geben hierüber die Protokolle an, daß es sich hier um das Fett sehr stark abgemagerter Tiere gehandelt habe. Auch meine übrigen Zahlen beziehen sich auf Tiere, bei welchen die Nahrungsaufnahme gestört war, die sich aber mehr oder minder von ihrem Körperfette ernähren mußten.

Wenn demnach von dem Tiere das Körperfett angegriffen wird, so werden in erster Linie oder vorwiegend jene Bestandteile angegriffen, welche die Jodzahl beeinflussen, also die Glyceride der ungesättigten Säuren (Ölsäure), während das Tripalmitin und Tristearin entweder erst später oder im geringen Ausmaße abgebaut werden.

Konnten einerseits für das Körperfett des Pferdes bestimmte Daten erhoben werden, so ist es nicht gut möglich, eine einheitliche Jodzahl für das Nahrungsfett zu ermitteln, weil ja ein Mischfutter gefüttert wurde, bestehend aus Hafer, Heu und Strohhäcksel. Bei dem Umstande jedoch, als das Fett des Heues und Strohes den Charakter des Pflanzenwachses zeigt, die Jodzahl seiner Fettsäuren gering ist, ist bezüglich der Jodzahl der Fettsäuren des Nahrungsfettes wohl das Fett des Hafers ausschlaggebend. Es kann also die Jodzahl des Hafers als Grenzzahl angesehen werden, unter welcher die dem Durchschnitte des Nahrungsfettes entsprechende Jodzahl der Fettsäuren liegen muß.

Roßmeißl $\left(^{8}\right)$ gibt die Jodzahl für die Fettsäuren des Haferöls mit 104,2 an.

Die Jodzahlen, welche sich für die Fettsäuren des Blutes gesunder Pferde gefunden haben, liegen nun in jenen Grenzen, welche einerseits durch die Jodzahl der Feltsäuren des Haferöls, 
anderseits durch die Jodzahlen der Fettsäuren des Körperfettes gesteckt sind, so daß die vorerwähnte Vorstellung von dem Transporte des Fetles auf dem Wege der Blutbahn der Grundlage nach plausibel erscheint. Eine Abweichung zeigt nur die Zahl $\mathrm{Nr}$. 24, welche sich auf ein rekonvalescentes Pferd bezieht, und diese Zahl soll später besprochen werden.

In gewöhnlichen tierischen Fetten, deren Fettsäuren fast ausschließlich aus Stearinsäure, Palmitinsäure und Ölsäure bestehen, ist für die Jodzahl der Fettsäuren die Menge der im Fett enthaltenen Ölsäuren maßgebend. Die Jodzahl reiner Ölsäure berechnet sich aus der Formel $\mathrm{C}_{18} \mathrm{H}_{32} \mathrm{O}_{2}$ mit 90,1 , das heißt für ein gewöhnliches tierisches Fett muß die Jodzahl der Fettsäuren kleiner sein als 90,1.

Nun stehen aber in der Tabelle für das Blut gesunder Tiere die Jodzahlen mehrfach hart an dieser Grenze. Da nicht angenommen werden kann, daß dieses Blutfett reines Triolein ist, und da einelne Zahlen sogar über 90,1 hinausgehen und sich der Zahl 104,2 (Haferöl) nähern, wobei von Nr. 24 ganz abgesehen wird, so erscheint durch diese hohen Zahlen dem Blutfett der Stempel des Nahrungsfettes aufgedrückt zu sein; es spricht dieser Umstand dafür, daß das Blutfett gesunder Pferde wenigstens hauptsächlich aus der Nahrung stammt und nicht durch Abbau des Körperfetts hineingekommen ist.

Was den Cholesteringehalt des normalen Blutes betrifft, so möchte ich die von mir erhobenen Zahlen auch nur mit einiger Reserve aufgenommen wissen. Die Digitonin-Methode nach Windaus zur quantitativen Bestimmung des Cholesterins ist an sich zwar eine bewährte, exakte Methode zur Bestimmung des Cholesterins. Da aber im Blute auch Veränderungsprodukte des Cholesterins, (0xycholesterine) möglicherweise auch andere Alkohole enthalten sind, welche durch Digitonin teilweise ausgefällt werden können oder die Fällbarkeit des Cholesterins beeinflussen könnten, so muß mit der Möglichkeit gerechnet werden, daß die gefundenen Werte von den wahren W'erten etwas abweichen. Man wird also bei Betrachtung der Resultate von kranken Pferden nur aus erheblichen Differenzen Schlüsse ziehen können. 
Uber das Felt des Blutes bei gesunden und kranken Pferden. 121

Noch unsicherer wird natürlich damit die Berechnung der Menge der anderen unverseif baren Substanzen. Trotzdem kann, wie schon erwähnt wurde, aus den bezüglichen Zahlen der Schluß gezogen werden, daß solche Substanzen vorbanden sind und da $B$ ihre Menge nicht allzu gering ist. Zur ungefähren Orientierung über die Frage, was etwa diese anderen unverseifbaren Substanzen sind, wurde die Jodzahl des gesamten unverseifbaren Rückstandes (Cholesterin + anderer unverseifbarer Substanz) ermittelt. Die Jodzahl des reinen Cholesterins wurde von Lewkowitsch(9) im Mittel mit 67,7 gefunden. Sie berechnet sich aus der Formel $\mathrm{C}_{27} \mathrm{H}_{44} \mathrm{O}$ mit 66 . Der Umstand, daß die Jodzahlen des unverseifbaren Rückstandes im Blute gesunder Pferde durchwegs kleiner sind als die Jodzahl des Cholesterins, besagt, daß die Jodzahl der anderen unverseifbaren Substanz erheblich kleiner sein muß, als die Jodzahl des Cholesterins, und legt, da diese anderen unverseifbaren Substanzen aus einem Gemenge verschiedener Stoffe bestehen, die Annahme nahe, daß zumindest ein Teil dieser Stoffe gar kein Jod addiert, also aus gesättigten Substanzen, wahrscheinlich gesüttigten höheren Alkoholen besteht. Weitere Schlüsse, etwa durch ziffernmäßige Abrechnung der Jodzahl, welche der ermittelten Cholesterinmenge entspricht, erscheinen, wie schon früher erwähnt wurde, nicht angängig.

Brustsouche.

\begin{tabular}{|c|c|c|c|c|c|c|c|}
\hline Nr. & Anmerkungen & $\begin{array}{c}\text { Trocken } \\
\text { substanz } \\
\text { in } g\end{array}$ & $\begin{array}{l}\text { Neu- } \\
\text { tralfett } \\
\text { in } g\end{array}$ & $\begin{array}{l}\text { Chole- } \\
\text { sterin } \\
\text { in } g\end{array}$ & $\begin{array}{c}\text { Andere } \\
\text { unver- } \\
\text { seifbare } \\
\text { Sub- } \\
\text { stanz } \\
\text { in } g\end{array}$ & $\begin{array}{c}\text { Jodzahl } \\
\text { der } \\
\text { Felt- } \\
\text { säuren }\end{array}$ & $\begin{array}{c}\text { Jodzald } \\
\text { der un- } \\
\text { verseif- } \\
\text { baren } \\
\text { Sub- } \\
\text { stanz. }\end{array}$ \\
\hline 7 & & 169,1 & - & - & 一 & - & - \\
\hline 8 & 1. Untersuchung & 182,2 & - & 一 & - & - & - \\
\hline 9 & 1. & 153,9 & 一 & - & - & - & 一 \\
\hline 10 & v. 8 . & 180,9 & - & - & 一 & - & 52,1 \\
\hline 11 & 1. & 175,8 & 5,01 & & 50 & 77,9 & 31,7 \\
\hline
\end{tabular}


Johann Rudolf,

Brustseuche (Fortsetzung).

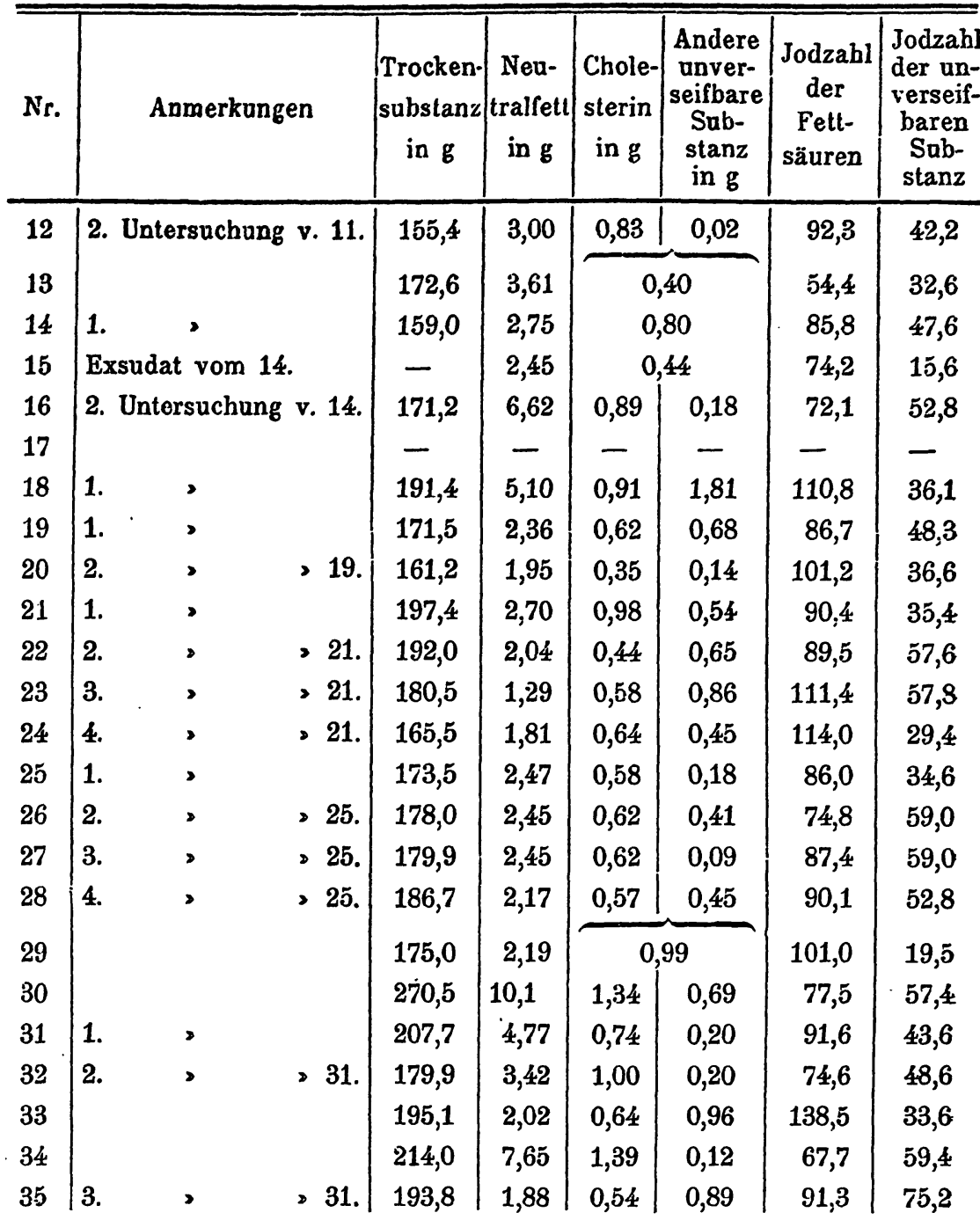

Vergleicht man die Zahlen für Neutralfett nur dieser Tabelle mit den bezüglichen Zahlen von gesunden Pferden, so finden sich bei den brustseuchekranken Pferden auch Werte, welche in denselben Rahmen fallen wie bei gesunden. Es kommen aber auch erheblich, oft ganz beträchtlich höhere, als. auch auffallend niedrige Werte vor. 
Mit den besonders hohen Werten für Neutralfett sind auch hohe Werte für Trockensubstanz verbunden. Nicht als ob hier eine Proportionalität bestehen würde; das Plus der Trockensubstanz ist auch nicht einzig und allein auf die größere Menge von Neutralfett zúrückzuführen. Es ist vielmehr in diesen Fällen gewiß eine Lipämie vorhanden und daneben auch eine Abnahme des Wassergehaltes, also eine Eindickung des Blutes.

Den Schlüssel zur Erklärung dieser außerordentlichen Mannigfaltigkeit der Neutralfettmengen bei Brustseuche ergeben jene Fälle, in welchen das Blut mehrmals im Krankheitsverlaufe untersucht wurde. In diesen Fällen sind die abnorm hohen Werte auch zeitlich immer die ersten. Im weiteren Verlauf der Krankheit sinken die Fettmengen und zwar allmählich bis unter die Norm und an dem Falle 21-24 wird der letzte Wert (24) wieder etwas höher, er nähert sich der Norm. Auch in jenem Stadium, in welchem die Pferde nach dem Resultate der klinischen Untersuchung als gesund gelten können, kann der Fettgehalt nicht zur Norm zurückgekehrt sein, er kann vielmehr unter der Norm liegen.

Eine Ausnahme macht der Fall 14-16, bei welchem ein erhebliches Ansteigen des Fettgehaltes im Verlaufe der Krankheit zu verzeichnen ist. Dieser Fall unterscheidet sich aber dadurch von den anderen Fällen, daß er zum Tode des Tieres führte, während jene in Heilung ausgingen. Überhaupt finden sich die großen Neutralfettmengen dort, wo schwere Krankheitserscheinungen vorhanden sind, während das $\mathrm{Ab}$ sinken der Neutralfettmengen unter die Norm als ein Symptom der Heilung aufzufassen ist.

In Fällen, welche klinisch leicht verlaufen, weichen die Neutralfettmengen kaum, oder nur wenig von der Norm ab. Daß die besonders hohen Werte keine physiologische Lipämie bedeuten können, geht schon daraus hervor, daß die schwerkranken Tiere wenig oder gar kein Futter aufnehmen und schlecht verdauen. Übrigens sind, wenn man die Gesamtmenge des Blutes des kranken Tieres berücksichtigt, die im gesamten Blute dieses Tieres enthaltenen Fettmengen, absolut 
genommien, weit größer als die Fettmenge mehrerer Kilogramm Hafer, und soviel nimmt ein schwerkrankes Pferd nicht an Hafer auf. Anderseits sinkt die Fettmenge im Blute gerade dann, wenn die Nahrungsaufnahme besser wird.

Die Jodzahlen der Fettsüuren sind bei brustseuchekranken Pferden vielfach nicht wesentiich anders als bei gesunden Pferden; es finden sich aber auch hier Werte, welche aus diesen Grenzen herausfallen.

Auch hier scheint sich aus jenen Fällen, in welchen das Blut mehrmals im Verlaufe der Krankheit untersucht wurde, eine Gesetzmäßigkeit zu ergeben. Man sieht im allgemeinen mit dem Fortschreiten der Rekonvalescenz ein Ansteigen der Jodzahl der Fettsäuren. Nur im Falle Nr. 14-16, der zum Tode führte, findet sich ein Abfallen. Wo unzulängliche Daten vorliegen, fällt der Wert anfangs und steigt bei eintretender Besserung.

Die Deutung dieser Tatsache dürfte im folgenden liegen. Im Anfange der Krankheit frißt das Pferd nicht oder nur wenig, es zehrt vielmehr von seinem Körperfette. Da nun, wie früher auseinandergesetzt wurde, vom Körperfett zuerst oder vorwiegend Triolein angegriffen wird, so muß auch in dieser Periode das Neutralfett des Blutes vorwiegend aus Triolein bestehen; die Jodzahl der Fettsäuren muß also der Jodzahl der Ölsäure $(90,1)$ mehr oder weniger nahe kommen und das stimmt mit der Tabelle im Beginn der Krankheit. In dem Maße nun, als der Vorrat an Triolein im Körperfett immer kleiner wird, müssen auch die Glyceride der gesättigten Fettsäuren, Tripalmitin und Tristearin, mit herangezogen werden und ins Blut gelangen. Die Jodzahl der Fettsäuren muß immer kleiner werden und wird sich, wenn die Zehrung vom Körperfette entsprechend lange dauert, jenen Werten nähern, die ich für das Körperfett kranker Tiere gefunden habe. So niedrige Werte finde ich tatsächlich, wie vorstehende Tabelle angibt.

Wenn nun der Krankheitsprozeß sich zum Bessern gewendet hat, so beginnt das Pferd allmählich zu fressen. In der Jodzahl der Fettsäuren des Blutes gewinnt daher die hohe .Jodzahl der Fettsäuren des Haferöls $(104,2)$ immer mehr Ein- 
Über das Fett des Blutes bei gessaden und kranken Pferden. 125

fluß; die Jodzahl der Fettsäuren des Blutes steigt daher allmählich wieder an.

Nun nimmt aber im weiteren Verlauf der Rekonvalescenz diese Jodzahl Werte an, welche über die Jodzahl der Fettsäuren des Hafers hinausgehen. Da weder in der Nahrung noch im Körper des Tieres sich Fettsäuren von solchen Jodzahlen finden, so bleiben nur zwei Annahmen übrig.

Die eine würde darin bestehen, daß der Körper anderswo, vieileicht im Blute selbst aus gesättigten Fettsäuren ungesättigte, also die einfache Bindung von Kohlenstoffatomen in eine doppelte verwandelt hat.

Nach der Gleichung<smiles>CC(C)C=COC(C)C</smiles>

ist dieser Prozeß ein Oxydationsprozeß, also ein Schritt zum Abbau, zur Verbrennung der gesättigten oder ungesättigten Fettsäuren.

Die Zunahme der Jodzahl könnte allerdings auch dadurch erklärt werden, daß man annimmt, die Ölsäure oder eine andere ungesättigte Säure würde durch Verkürzung ihrer Kohlenstoffkette zu einer Säure von geringerem Molekulargewichte. Die letztere Annahme wird dadurch unwahrscheinlich, wenn wir bedenken, daß in jeder ungesättigten Verbindung die doppelt gebundenen Kohlenstoffatome der locus minoris resistentiae sind und daß die ermittelte Jodzahl sich auf wasserunlösliche, nicht flüchtige Fettsäuren bezieht, während durch Verkleinerung des Molekulargewichtes die Löslichkeit einer Fettsäure im Wasser und ihre Flüchtigkeit immer mehr zunimmt. Die erste Annahme wird dadurch zur wahrscheinlicheren.

Ob der Prozeß, den die Jodzahl andeutet, schon normalerweise sich vollzieht und ob er eine Reaktion ist, die der Tierkörper während der Rekonvalescenz erst gelernt hat in Analogie zu den Anschauungen Abderhaldens über die Entstehung von Körperfermenten, das sind Fragen, deren Beantwortung ich dahingestellt sein lassen muß.

Hoppe-Seyler's Zeitschrift f. physiol. Chemie. CI. 
Nicht die gleiche RegelmäPigkeit wie beim Neutralfette kann ich im Wechsel des Cholesteringehaltes finden. Ich finde insbesondore die Angaben von Bacmeister und Haves, sowie von Henes, daß bei fieberhaften Krankheiten der Cholesteringehalt des Blutes vermindert ist, bei der Brustseuche der Pferde nicht bestätigt. Ich habe früher schon darauf hingewiesen, daß der Pflanzenfresser sich vom Fleischfresser im Cholesterinstoffwechsel darin unterscheidet, daß er sich sein Cholesterin erst bilden muß.

Petechialfieber.

\begin{tabular}{|c|c|c|c|c|c|c|c|}
\hline $\mathrm{Nr}$. & Anmerkungen & $\begin{array}{c}\text { Trocken } \\
\text { substanz } \\
\text { in } \mathbf{g}\end{array}$ & $\begin{array}{c}\text { Neu- } \\
\text { tralfett } \\
\text { in } g\end{array}$ & $\begin{array}{c}\text { Chole- } \\
\text { sterin } \\
\text { in } g\end{array}$ & $\begin{array}{l}\text { Andere } \\
\text { unver- } \\
\text { seifbare } \\
\text { Sub- } \\
\text { stanz } \\
\text { in } g\end{array}$ & $\begin{array}{c}\text { Jodzahl } \\
\text { der } \\
\text { Fett- } \\
\text { säuren }\end{array}$ & \begin{tabular}{|} 
Jodzahl \\
der an- \\
verseif- \\
baren \\
Sub- \\
stanz
\end{tabular} \\
\hline 36 & & 215,4 & 3,51 & \multicolumn{2}{|c|}{1,27} & 96,5 & 48,9 \\
\hline 37 & & 199,7 & 3,50 & \multicolumn{2}{|c|}{0,71 . - } & 78,5 & 50,4 \\
\hline 38 & & 212,4 & 4,05 & \multicolumn{2}{|c|}{0,49} & 86,1 & 52,9 \\
\hline 39 & & 189,4 & 3,12 & 0,49 & 0,50 & 86,5 & 52,8 \\
\hline 40 & 1. Untersuchung & 202,5 & 2,51 & 0,62 & 0,55 & 98,0 & 53,7 \\
\hline 41 & v. 40. & 136,8 & 2,64 & 0,47 & 0,43 & 96,3 & 59,7 \\
\hline
\end{tabular}

Die hier verzeichneten Zahlen unterscheiden sich mit Ausnahme der Probe 41 nicht wesentlich von den Zahlen, die bei gesunden Pferden gefunden wurden, was um so auffälliger ist, als das Petechialfieber eine schwere Allgemeinerkrankung darstellt. Es zeigt dies, daß die Veränderungen im Blute, welche bei der Brustseuche gefunden worden sind, nicht lediglich der Ausdruck gestörten Allgemeinbefindens sind, sondern jedenfalls auch mit der Natur des Krankheitsprozesses zusammenhängen.

Bei der Probe 41 fällt die geringe Ménge der Gesamttrockensubstanz auf, sie beweist, daß eine Hydrämie bestanden hat. Die Zahlen für Neutralfett, Cholesterin und «Andere unverseifbare Substanzen s sind daher nicht direkt vergleichbar mit den bezüglichen Zahlen der anderen Blutproben; es muß vielmehr die Hydrämie mitberücksichtigt werden. Dement- 
Uber das Felt des Blutes bei gesunden und kranken Pferden. 127

sprechend würde der Wert für Neutralfett von 2,64 $\mathrm{g}$ bis gegen $4 \mathrm{~g}$ rücken und sich bei diesem Falle, wie im Falle 14-16, in der Brustseuchetabelle eine Verschlimmerung des Krankheitsprozesses auch durch eine Erhöhung des relativen Fettgehaltes dokumentieren, wenn auch nicht in dem Grade, wie bei der Brustseuche.

Was die Jodzahl betrifft, so liegen hier greifbare Anhaltspunkte nicht vor, doch gelingt es unschwer mit Hilfe der Anschauungen, welche bei der Brustseuche entwickelt wurden, auch diese Zahlen zu deuten.

Krankheiten des Verdaungstraktes.

\begin{tabular}{|c|c|c|c|c|c|c|c|}
\hline $\mathrm{Nr}$. & Anmerkungen & $\begin{array}{c}\text { Trocken- } \\
\text { substanz } \\
\text { in } g\end{array}$ & $\begin{array}{c}\text { Neu- } \\
\text { tralfett } \\
\text { in } g\end{array}$ & $\begin{array}{c}\text { Chole- } \\
\text { sterin } \\
\text { in } g\end{array}$ & $\begin{array}{l}\text { Andere } \\
\text { unver- } \\
\text { seifbare } \\
\text { Sub- } \\
\text { stanz } \\
\text { in g }\end{array}$ & $\begin{array}{c}\text { Jodzahl } \\
\text { der } \\
\text { Fett- } \\
\text { säuren }\end{array}$ & $\begin{array}{c}\text { Jodzahl } \\
\text { der un- } \\
\text { verseif- } \\
\text { baren } \\
\text { Sub- } \\
\text { stanz }\end{array}$ \\
\hline 42 & & 189,0 & 3,10 & \multicolumn{2}{|c|}{0,71} & 86,5 & 80,0 \\
\hline 43 & 1. Untersuchung & 176,0 & 2,43 & 0,77 & 1,37 & 87,6 & 36,8 \\
\hline 44 & v. 43. & 217,2 & 6,33 & 0,96 & 0,64 & 80,1 & 50,5 \\
\hline 45 & & 200,0 & 8,32 & 1,07 & 1,72 & 95,9 & 37,9 \\
\hline 46 & & 195,5 & 2,54 & 0,95 & 1,12 & 99,1 & 56,3 \\
\hline 47 & & 239,5 & 3,06 & 0,87 & 0,87 & 114,0 & 47,5 \\
\hline
\end{tabular}

In der vorstehenden Tabelle finden sich zunächst zwei hohe Zahlen für Neutralfett, nämlich bei den Proben 44 und 45. Diese Proben stammen von Pferden, welche zur Zeit der Blut. entnahme an Diarrhoe litten, während bei den anderen Blutproben, wo keine Diarrhoe festzustellen war, die Neutralfettmengen nicht erhöht sind. Daß die Lipämie der Proben 44 und 45 nicht als physiologisch zu deuten ist, liegt auf der Hand, da ja bekannterweise Diarrhoe eine schlechte Resorption der Nährstoffe durch den Darm bedingt. Es macht den Eindruck, als ob die Ernährung des Körpers auf dem Wege der Resorption vom Verdauungstrakte aus gestört ist und der Organismus von seinem Körperbestande zehren muß und darum zuerst die Fettdepots angreift. Der Organismus 
würde sich also hier hauptsächlich auf Kosten seines Körperfettes erhalten, während sonst die Kohlenhydrate der Nahrung seinen Bedarf an Nährstoffen decken.

$\mathrm{Daß}$ diese Lipämie nicht auf dieselbe Stufe zu stellen ist, wie dio Lipämie bei der Brustseuche, beweist die Probe 47. Sie stammt von einem Pferde kurz vor dem Tode und zeigt doch keine Lipämie.

Auffallend sind in der vorstehenden Tabelle die relativ hohen Zahlen für Cholesterin. Sie sind um so auffälliger, weil auch die Zahlen für sAndere unverseifbare Substanzen. im allgemeinen erheblich höher sind, als beim normalen Blute. Wollte man daher auch die Genauigkeit der Windausschen Digitonin-Methode für das Blut nicht anerkennen, so würden die hohen Werte für den unverseifbaren Rückstand (Cholesterin + Anderer unverseifbaren Substanzen) jedenfalls ihre Zuverlässigkeit beweisen. Für mich besteht übrigens kein Grund, die Genauigkeit der Digitoninmethode soweit anzuzweifeln, daß sie dieses Beweises erst bedürfte.

Der Umstand wurde deshalb besonders betont, weil die vorstehenden Cholesterinbefunde allem Anscheine nach im direkten Widerspruch stehen mit den Angaben Bacmeisters und Haves, die angeben, daß der Cholesteringehalt des Blutes bei verminderter Nahrungsaufnahme abnimmt. Da Erkrankungen des Darmkanals in der Regel eine Störung der Verdauung und Resorption bedingen, so verursachen sie an sich schon eine verminderte Aufnahme von Nährstoffen auch dann, wenn das Pferd Futter aufnimmt, und trotzdem enthält das Blut relativ viel Cholesterin bezw. unverseifbaren Rückstand.

Eine befriedigende Erklärung für diese hohen Zahlen zu geben, bin ich nicht imstande; doch liegt es nahe, an den Übertritt von Gallenbestandteilen in das Blut zu denken, bezw. eine gestörte Ausscheidung von Cholesterin durch die Galle, vielleicht sogar an die Umwandlung der durch die Galle nicht ausgeschiedenen Gallensäuren zu Cholesterin und diesem verwandten Stoffen.

Unter den Jodzahlen der Fettsäuren finden sich solche, welche der Jodzahl der Ölsäüre nahe kommen $(42,43,44)$. 
Über das Fett des Blutes bei gesunden und kranken Pferden. 129

Sie deuten, wie schon früher auseinandergesetzt wurde, darauf, daß der Körper von seinem eigenen Fette zehrt. Mit diesen Auseinandersetzungen stimmt auch überein, daß in den Proben 43 und 44 die später erhobene Zahl (Nr. 44) kleiner ist. Die beiden Tiere fressen auch, wie die Versuchsprotokolle anführen, schlecht.

Die Proben 45 und 46 zeigen Jodzahlen, welche höher sind und zwischen den bezüglichen Zahlen der Ölsäure und des Haferöls liegen. Die Versuchsprotokolle besagen, daß diese beiden Tiere gut gefressen haben. Es wäre also denkbar, daß trotz der allgemeinen Verdauungsstörungen doch etwas von dem leicht verdaulichen Fette des Hafers resorbiert wurde und seinen Einfluß auf die Jodzahlen der Fettsäuren des Blutes ausübte. Diese Vermutung steht bei Nr. 45 nicht im Widerspruche mit der früher gegebenen Deutung der Lipämie in diesem Falle, denn sie gründet sich auf eine mangelhafte Verdauung und Resorption der Kohlenhydrate, während hier von einer Resorption des leicht verdaulichen Haferfettes die Rede ist, welche trotz der gestörten Kohlenhydratverarbeitung denkbar wäre.

Die Probe 47 endlich, bei welcher eine die Jodzahl der Fettsäuren des Haferöls übersteigende Jodzahl gefunden wurde, bezieht sich auf ein Tier mit raschem Krankheitsverlaufe vor dem Tode, bei welchem naturgemäB die allgemein pathologischen Verhältnisse nicht rasch überblickt werden können.

Tetanus.

\begin{tabular}{c|c|c|c|c|c|c|c}
\hline \hline Nr. & Anmerkung & $\begin{array}{c}\text { Trocken- } \\
\text { substanz } \\
\text { in } g\end{array}$ & $\begin{array}{c}\text { Neu- } \\
\text { tralfett } \\
\text { in g }\end{array}$ & $\begin{array}{c}\text { Chole- } \\
\text { sterin } \\
\text { in } \mathbf{g}\end{array}$ & $\begin{array}{c}\text { Andere } \\
\text { unver- } \\
\text { seifbare } \\
\text { Sub- } \\
\text { stanz } \\
\text { in g }\end{array}$ & $\begin{array}{c}\text { Jodzahl } \\
\text { der } \\
\text { Fett- } \\
\text { säuren }\end{array}$ & $\begin{array}{c}\text { Jodzahl } \\
\text { des un- } \\
\text { verseif- } \\
\text { baren } \\
\text { Rück- } \\
\text { standes }\end{array}$ \\
\hline 48 & & 230,8 & 3,78 & 1.04 & 0,76 & 90,9 & 33,3 \\
\hline
\end{tabular}

Es ist natürlich nicht angebracht, aus einem einzigen Falle bindende Schlüsse anf den Chemismus beim Tetanus zu ziehen. Ich will daher den Befund nur kurz diskutieren. Die. 
hohe Zahl für 'Trockensubstanz beweist eine Eindickung des Blutes, welche durch die gesteigerte Schweißsekretion leicht erklärt werden kann. Lipämie ist nicht vorhanden. Die Jodzahl der Fettsäuren liegt in normalen Grenzen und steht vielleicht zufällig der Jodzahl der Ölsäure sehr nahe. Auffällig ist der relativ hohe Gehalt an Cholesterin und cAnderer unverseifbaren Substanzen.

Wenn ich nun zum Schlusse noch auf die eingangs gestellte allgemeine Frage zurückkomme, ob die fettartigen Substanzen des Blutes kranker Pferde Unterschiede zeigen gegenüber jenen gesunder Tiere, so kann diese Frage auf Grund vorstehender Untersuchungsresultate nur bejaht werden. Es kann auch gesagt werden, daß die Art und der Grund vorstehender Unterschiede, wenn man sie gerade auch nicht spezifisch für die einzelnen Krankheiten nennen kann, doch mit den der Krankheit eigentümlichen Veränderungen im organischen Zusammenhange stehen und daß verschiedene Krankheiten auch verschiedene Veränderungen in den Fettsubstanzen hervorrufen und zwar auch Krankheiten, welche man nicht als Stoffwechselkrankheiten $\mathrm{zu}$ bezeichnen pflegt.

\section{Literatur.}

1. Deutsche Medizinische Wochenschrift, 1914.

2. Hoppe-Seylers Zeitschrift für Physiologische Chemie, Bd. 54 .

3. Unna-Festschrift, Bd. II, 1910.

4. Deutsches Archiv für klinische Medizin, Bd. 111, 1. und 2. Heft.

5. Abderhalden, Biochemische Arbeitsmethoden, Bd. 5.

6.

7.u.9. Benedikt-Ulzer, Analogie der Fette und Wachsarten.

8. Wiener tierärztliche Monatsschrift, I. Jahrg. 